\title{
Distribución potencial de los bosques de Prosopis flexuosa en la Provincia Biogeográfica del Monte (Argentina)
}

\author{
Mariana Perosa ${ }^{1}$; Facundo Rojas ${ }^{2, \varpi}$; Pablo Villagra ${ }^{2,3}$; Marcelo F. Tognellit; Rodolfo \\ Carrara $^{5}$ \& Juan A. Alvarez ${ }^{2,3}$
}

1. Instituto de Ciencias Ambientales-Universidad Nacional de Cuyo. Mendoza, Argentina. 2. Instituto Argentino de Nivología, Glaciología y Ciencias Ambientales. IANIGLA. CCT Mendoza, CONICET. 3. Facultad de Ciencias Agrarias. Universidad Nacional de Cuyo. Mendoza, Argentina. 4. IUCN - CI Biodiversity Assessment Unit, Conservation International. Arlington, USA. 5. Instituto Argentino de Investigaciones de las Zonas Áridas. IADIZA. CCT Mendoza, CONICET.

\begin{abstract}
Resumen. En las zonas áridas de Argentina, los bosques de Prosopis flexuosa son de suma importancia porque protegen el suelo en áreas con riesgo elevado de desertificación, y porque ofrecen diversos recursos a los pobladores locales (e.g., leña, forraje y productos alimenticios derivados de sus frutos). La actividad antrópica ha tenido un fuerte impacto sobre estos bosques, en especial desde la segunda mitad del siglo XIX. Por lo tanto, conocer su distribución potencial y compararla la distribución actual permitirá definir zonas prioritarias para la conservación y proponer posibles áreas de reforestación. El objetivo de esta investigación fue generar un modelo de distribución potencial de los bosques de P. flexuosa en la Provincia Biogeográfica del Monte. Para obtener el mapa modelo se utilizó el software Maxent, tomando como datos los registros georeferenciados de presencia de bosques de P. flexuosa y de variables ambientales relevantes para la distribución de muchas especies (variables climáticas, subórdenes de suelos y profundidad de napa freática). En general, el mapa de distribución potencial resultante concuerda con la ubicación de los principales valles, bolsones y llanuras del Monte, en donde se conoce la existencia actual de los bosques de $P$. flexuosa. Sin embargo, las superficies y los límites de los bosques estimados y los observados difieren en algunos casos, lo que se relacionaría con disturbios antrópicos y/o con otras variables no consideradas en el modelo, como la probabilidad de incendios.
\end{abstract}

[Palabras clave: modelos, biogeografía, algarrobal, zonas áridas]

\begin{abstract}
Aвstract. Potential distribution of Prosopis flexuosa woodland in the Monte desert (Argentina): In arid areas of Argentina, Prosopis flexuosa woodlands are of foremost importance since they are thought to protect the soil against erosion in a region with a high risk of desertification, and because they offer several resources to local inhabitants, such as firewood, forage for cattle, and food products derived from its fruit. These woodlands have been strongly impacted by human activities, mainly during the first half of the XIX ${ }^{\text {th }}$ century. Knowledge about their potential distribution, as well as a comparison between potential and actual distributions, would allow priority areas for conservation and possible areas for reforestation to be defined. The aim of this work was to generate a model of the potential distribution of P. flexuosa woodland within a region of arid shrublands in central Argentina, known as the Monte desert. Maxent software was used to generate the distribution models. The sofware uses as input a set of georeferenced data of the presence of $P$. flexuosa woodland, and of those relevant for the distribution of species (e.g., climatic, altitude, slope, aspect, soil and water table depth). The resulting potential distribution model generally coincided with the main valleys, depressions and plains of the region, where $P$. flexuosa woodland are known to exist currently. However, area and limits of observed and estimated woodland patches differ in some cases, which could be related with anthropogenic disturbances in some of the patches or with variables not included in the model, such as the probability of wildfires.
\end{abstract}

[Keywords: models, biogeography, algarrobal, arid lands]

\section{INTRODUCCIÓN}

Los cambios provocados por las actividades humanas en la cubierta vegetal han producido modificaciones significativas en la estructura, composición de especies y distribución de los bosques en el mundo. En este sentido, la Organización de las Naciones Unidas (ONU) advirtió sobre los problemas que ocasionan los millones de hectáreas de bosques que se deforestan por año en el mundo, teniendo en cuenta el impacto sobre los servicios

Editora Asociada: Adriana Ruggiero

$\triangle$ frojas@mendoza-conicet.gob.ar ambientales que brindan: conservación del suelo, calidad del agua, regulación hídrica y fijación de emisiones de gases de efecto invernadero, entre otros (Cumbre de Río 1992; FAO 2011). En Argentina, en los últimos años, la deforestación ha superado las 200000 ha/año (Montenegro et al. 2007) debido principalmente a la expansión creciente de la frontera agropecuaria, en especial del cultivo de soja; en este sentido, la región chaqueña y el noroeste argentino son las zonas más afectadas. Sin embargo, los cambios en el 
Monte han sido débilmente estudiados (Grau et al. 2007; Zarrilli 2008). Esta problemática ha generado grandes debates que derivaron en la sanción de la Ley Nacional N² 26331 de Protección de los Bosques Nativos, sancionada en noviembre de 2007 y reglamentada en febrero de 2009. La norma busca frenar la tala indiscriminada de los bosques y establece entre sus artículos una moratoria a los desmontes por cinco años, o hasta que cada provincia desarrolle un ordenamiento territorial en sus bosques nativos. También presenta criterios de sustentabilidad para el cuidado, aprovechamiento, protección y manejo adecuado de los mismos. Esta ley refleja la necesidad de estudios que determinen las condiciones y características de los bosques, su distribución, el grado de amenaza al que están sometidos y el tipo de manejo aplicado.

Los bosques de P. flexuosa son de suma importancia al ser considerados bosques protectores del suelo en una zona de alto riesgo de desertificación, y porque ofrecen diversos recursos a los pobladores locales tales (e.g., leña, forraje y productos alimenticios derivados de sus frutos). Aun así, a lo largo de la historia no han captado tanta atención como otras áreas boscosas debido a que presentan índices de diversidad relativamente bajos $\mathrm{y}$, desde un punto de vista económico, una menor productividad de recursos naturales. Sin embargo, la presencia de numerosos endemismos que representan los extremos adaptativos de los diferentes grupos taxonómicos al estrés hídrico, y la alta fragilidad y la baja capacidad de regeneración de estos sistemas convierten a estas áreas en zonas prioritarias de conservación (Shmida 1985; Villagra et al. 2004).

La distribución actual de los bosques de $P$. flexuosa en el Monte no sólo es el resultado de las condiciones naturales de esta región sino también de los diferentes usos y aprovechamientos del bosque. Numerosos trabajos explican cómo los algarrobales del Monte han sido fuertemente impactados por la actividad antrópica (Roig 1993; Abraham \& Prieto 1999; Prieto et al. 2003; Villagra et al. 2009; Rojas et al. 2009). Por esto resulta de suma utilidad determinar su distribución potencial para contrastarla con la distribución actual, histórica y con su uso. De esta manera se podría relacionar la eventual variación en la distribución con procesos de degradación, como así también definir zonas prioritarias para la conservación y reforestación. Los modelos de distribución potencial de especies determinan aquellas áreas con las condiciones ambientales más adecuadas para el desarrollo de poblaciones de una especie concreta o de una determinada formación vegetal, a partir de una serie de variables ambientales y un conjunto de datos de presencia tomados a campo (Ferrier \& Guisan 2006; Tarkesh \& Jetschke 2012). Estos métodos han ganado popularidad en la última década y se han usado para responder preguntas en ecología, biogeografía, evolución, cambio climático, así como también en aplicaciones de conservación (Guisan \& Zimmermann 2000; Stauffer 2002; Guisan \& Thuiller 2005; Ferrier \& Guisan 2006; Richards et al. 2007; Schröder 2008; Elith \& Leathwick 2009; Kearney \& Porter 2009).

Para el caso de Sudamérica, son cada vez más abundantes los trabajos con diferentes objetivos y metodologías; en ellos se analizan diversas especies y con escalas geográficas variables (e.g., Naoki et al. 2006; Corbalán \& Debandi 2007; Ferrer et al. 2007; Giovanelli et al. 2008; Tognelli et al. 2009; Jayat et al. 2009; Torres \& Jayat 2010). En el Monte se han llevado a cabo estudios sobre la distribución actual de los bosques de Prosopis en algunos valles y bolsones (González Loyarte et al. 2000; Alvarez et al. 2006; Cesca 2011; Villagra et al. datos no publicados). Sin embargo, no existen estudios que estimen las distribuciones potenciales de los bosques de algarrobo para esta región. El objetivo de este trabajo es generar un modelo de distribución potencial de los bosques de $P$. flexuosa en el Monte desde $\operatorname{los} 25^{\circ}$ a $\left.\operatorname{los} 36^{\circ} \mathrm{S}\right)$.

\section{Materiales y Métodos}

\section{Área de estudio}

La Provincia Biogeográfica del Monte abarca $460000 \mathrm{~km}^{2}$ de la zona árida templada de la República Argentina. Se extiende desde los $24^{\circ} 35^{\prime}$ hasta los $44^{\circ} 02^{\prime} \mathrm{S}$ y desde los 62 $54^{\prime}$ a los $69^{\circ} 50^{\prime} \mathrm{W}$. El clima del Monte es semiárido a árido, con una media anual de precipitación inferior a $350 \mathrm{~mm}$. Las temperaturas medias varían entre $13.4^{\circ} \mathrm{C}$ en Trelew y $17.5^{\circ} \mathrm{C}$ en Tinogasta, y presenta una gran amplitud térmica anual (Morello 1958; Cabrera 1976; Labraga \& Villalba 2009). El área de estudio comprende la zona correspondiente al Monte de las Provincias de Salta, Tucumán, Catamarca, La Rioja, San Juan y Mendoza, y se extiende desde los $25^{\circ}$ hasta los $37^{\circ} \mathrm{S}$.

La vegetación del Monte se caracteriza por una estepa arbustiva con predominio de especies de 
la familia Zigophyllaceae (Larrea spp. y Bulnesia retama), las estepas edáficas de arbustos halófitos como Suaeda divaricata, Atriplex spp, Allenrolfea vaginata y el bosque dominado por especies del género Prosopis (Morello 1958; Cabrera 1976; Villagra et al. 2004). El bosque de Prosopis spp, conocido como "algarrobal", se desarrolla en zonas con una provisión extra de agua en profundidad donde funcionan como freatófitos (Jobbágy et al. 2011). El estrato arbóreo es muy abierto, dominado por $P$. flexuosa (especie más común y dominante en la mayor parte de la superficie boscosa) o por $P$. chilensis (en las márgenes de cauces de agua), acompañados por Geoffroea decorticans "chañar"; Capparis atamisquea "atamisqui" y especies del género Larrea "jarillas". Rossi \& Villagra (2003) destacan el valor de las especies del género Prosopis en el hábitat de otros organismos al generar heterogeneidad espacial y modificar la distribución espacial de las especies de los estratos arbustivos y herbáceos.

\section{Modelado de nicho para determinar la distribución potencial del bosque nativo}

Para obtener la distribución potencial de los bosques de $P$. flexuosa en el área de estudio se utilizó el programa Maxent versión 3.3.3k (Phillips et al. 2006; Elith et al. 2011; "http:/ / www.cs.princeton.edu/ schapire/maxent"). Se eligió esta técnica de modelado de nicho porque sólo requiere datos de presencia, y en comparación con otras técnicas ha probado tener un muy buen rendimiento, aun con bajo número de localidades de presencia (Hernández et al. 2006; Elith et al. 2006, 2010, 2011; Tognelli et al. 2009). Esta técnica ha sido muy utilizada para modelar la distribución potencial de especies individuales, y más recientemente ha demostrado su potencialidad en el modelado de las zonas donde puede existir una determinada comunidad vegetal (Tarkesh \& Jetschke 2012).

La precisión del modelo fue evaluada de acuerdo al valor de AUC (área bajo la curva) de las curvas ROC (característica operativa del receptor). Estas curvas se obtienen a partir de los valores de sensitividad (i.e., la tasa de verdaderos positivos) y de especificidad (i.e., la tasa de falsos positivos) del modelo (Phillips et al. 2006). Al ser Maxent un modelo que sólo utiliza datos de presencia, los valores de especificidad no son obtenidos de datos de verdadera ausencia sino de datos aleatorios generados por el modelo en el área de estudio (que el programa denomina puntos de fondo: "background points"). Debido a estas características, el modelo hace predicciones sobre la base de muestras de instancia positiva (localidades de presencia) y muestras de instancia negativa (puntos de fondo). Con esta información se construyen las curvas ROC, que deben ser interpretadas en el contexto de pseudo-ausencias (ver Wiley et al. 2003).
Maxent, además, tiene la ventaja de que permite utilizar variables predictoras, tanto continuas como categóricas, y estima la importancia relativa de cada variable en la distribución de la especie mediante el procedimiento Jackknife (Guisan \& Zimmermann 2000). Este procedimiento permite evaluar el aporte de cada variable a la ganancia total del modelo. En este último caso, el software realiza dos tipos de procedimientos. En el primero se generan modelos sucesivos en los que se va extrayendo una variable por vez, para ver cómo disminuye la ganancia total de modelo al sustraer una variable determinada. En el segundo se generan modelos con sólo una variable por vez para ver cuánta información posee cada una y para comparar la ganancia con el modelo completo.

\section{Compilación de datos de presencia de bosque y selección de variables independientes}

Los puntos de presencia se obtuvieron a partir de estudios previos y en curso relacionados a la distribución y al estado de conservación de bosques de P. flexuosa (Cony 1993; Cesca et al. 2004; Álvarez et al. 2006; Villagra et al. datos no publicados). Esos puntos fueron tomados con GPS en diferentes valles y bolsones del Monte (Figura 1). Como el objetivo del presente trabajo es generar un modelo de distribución potencial de bosques de $P$. flexuosa y no de la especie en forma individual, no se tuvieron en cuenta puntos de presencia de árboles individuales sino de bosques de $P$. flexuosa; se consideró bosque a aquellas unidades de vegetación donde la densidad de individuos de porte arbóreo de $P$. flexuosa fue superior a 20 individuos/ha y su cobertura fue superior a $15 \%$. No se tuvieron en cuenta zonas con presencia de $P$. flexuosa de porte arbustivo.

Se utilizaron variables climáticas, edáficas e hídricas como variables ambientales predictoras (Tabla 1). Las variables climáticas se obtuvieron del sitio WorldClim (http:/ / www.worldclim.org), con una resolución de 30 segundos $\left(\sim 1 \mathrm{~km}^{2}\right)$ y corresponden al período 1950-2000. Estas variables fueron generadas a partir de interpolaciones de valores mensuales de temperatura y precipitación de estaciones meteorológicas (Hijmans et al. 2005). Estas variables se denominan bioclimáticas y son las que por lo general se utilizan en el modelado del nicho ecológico (ver Hijmans et al. 2005).

Para seleccionar las variables más relevantes se utilizaron los resultados de Perosa (2010), trabajo en el que las variables que tuvieron mayor contribución al modelo (entre 25 variables tenidas en cuenta para evaluar sus contribuciones a la existencia de algarrobales) fueron: temperatura media del trimestre más frío, amplitud térmica diaria, profundidad de la napa freática, subórdenes de suelos, precipitación anual y precipitación del trimestre más frío. Esto, sumado a la evaluación del aporte de cada variable a la ganancia total del modelo (Jackknife), en el que resultaron más importantes la temperatura mínima del mes más frío, la napa freática y los subórdenes de suelos. 


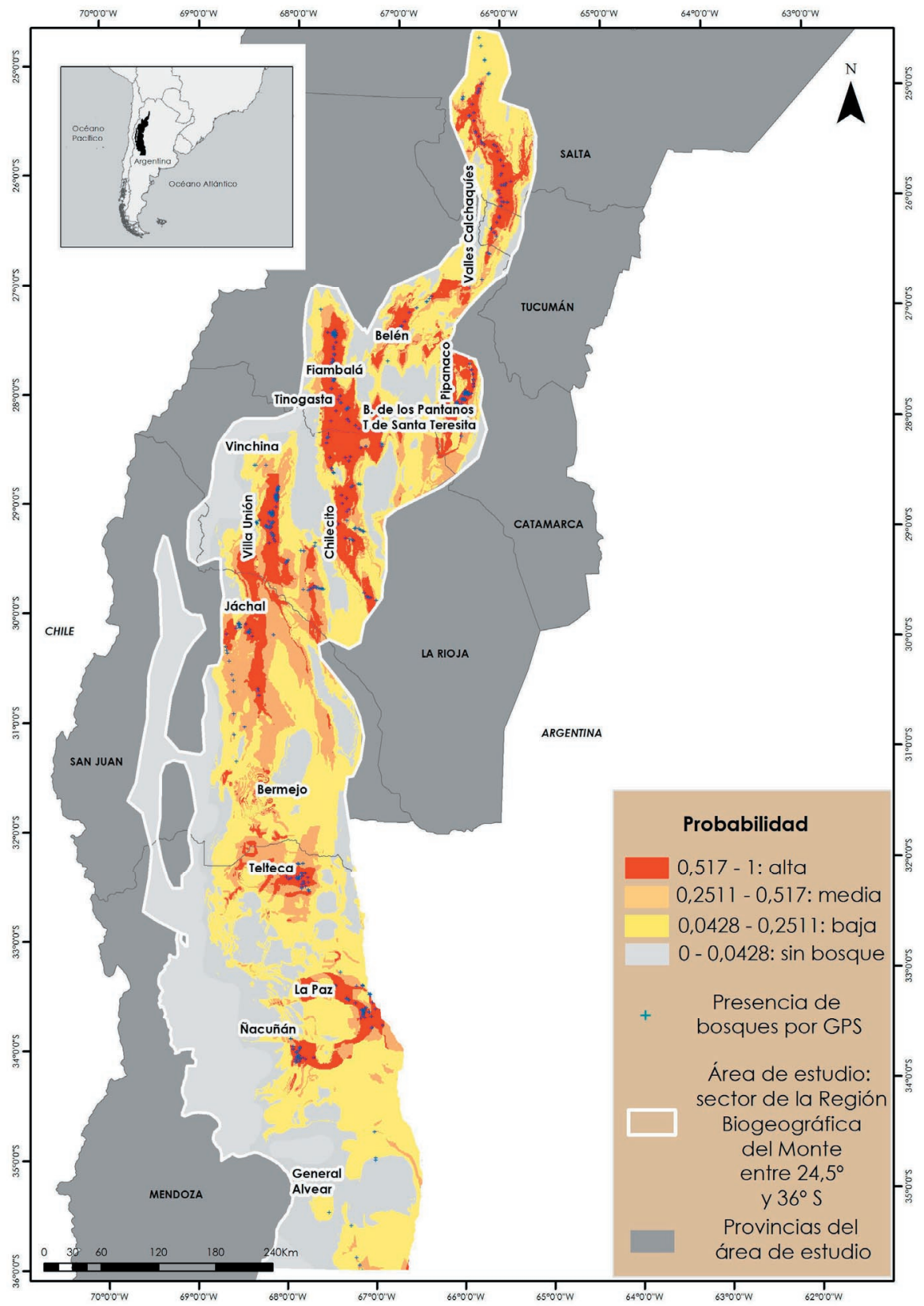

Figura 1. Distribución potencial de los bosques de Prosopis flexuosa en el Monte. Diferentes colores indican áreas con diferente probabilidad de ocurrencia de bosques en función de las características ambientales. Se incluyen los nombres de los bolsones y zonas donde se desarrollan los bosques y las localidades mencionadas en el texto.

Figure 1. Potencial distribution of Prosopis flexuosa woodlands in the Monte desert. Different colors indicate areas with different probabilities of woodland occurrences as a function of the environmental features. The names of the basins where woodlands occurs and the locations mentioned in the text.

La elección de estas variables respondió también a su importancia para la existencia de algarrobales, a partir de los estudios sobre ecología y fisiología de los respectivos bosques (Morello 1958; Roig 1985; Villagra et al. 2004; 2011; Jobbágy et al. 2011).

La profundidad de la capa freática se obtuvo recopilando la información de diferentes trabajos (Sosic 1971; Victoria et al. 1995; Rodríguez 1999; Asociación Argentina de Amigos de la Universidad
Ben Gurion 2006) y del Centro Regional de Aguas Subterráneas (CREA-San Juan). Para elaborar la base cartográfica de freática se utilizaron los datos digitalizados y georreferenciados facilitados por el CREA-San Juan, que fueron combinados con información obtenida para las provincias de Catamarca, La Rioja, Tucumán y Salta (en papel). Los mapas de agua subterránea en papel fueron digitalizados y georeferenciados mediante el software libre Kosmo GIS. Para la variable edáfica 
Tabla 1. Contribución relativa (\%) y aporte exclusivo de cada variable utilizada en el modelo.

Table 1. Relative contribution (\%) and unique contribution of each variable used in the model.

\begin{tabular}{|c|c|c|c|c|c|}
\hline Tipo de variable & Variable & $\begin{array}{c}\text { Contribución } \\
\text { al modelo } \\
(\%)\end{array}$ & $\begin{array}{c}\text { Aporte exclusivo } \\
\text { de cada variable } \\
\text { aislada }\end{array}$ & $\begin{array}{l}\text { Diferencia en } \\
\text { la capacidad } \\
\text { explicativa * }\end{array}$ & Fuente \\
\hline \multirow{4}{*}{$\begin{array}{l}\text { Variables } \\
\text { climáticas }\end{array}$} & Temperatura media & 23.7 & 0.41 & 1.09 & WordClim \\
\hline & $\begin{array}{l}\text { del trimestre más frio } \\
\text { Precipitación anual }\end{array}$ & 8.4 & 0.14 & 0.95 & \\
\hline & $\begin{array}{c}\text { Amplitud térmica } \\
\text { diaria }\end{array}$ & 6.9 & 0.15 & 0.95 & \\
\hline & $\begin{array}{l}\text { Temperatura mínima } \\
\text { del mes más frío }\end{array}$ & 5.6 & 0.26 & 0.84 & \\
\hline Variable edáfica & $\begin{array}{l}\text { Subórdenes de suelos } \\
\text { (v. categórica) }\end{array}$ & 11.8 & 0.19 & 0.89 & INTA \\
\hline Variable hídrica & Profundidad de napa & 7.2 & 0.11 & 0.96 & $\begin{array}{l}\text { SSRH-INA; Sosic 1971; } \\
\text { Rodríguez 1999; Asociación } \\
\text { Argentina de Amigos de } \\
\text { la Universidad Ben Gurion } \\
\text { 2006; Victoria et al. } 1995\end{array}$ \\
\hline
\end{tabular}

* medido por ganancia de entrenamiento regularizada

se utilizaron los subórdenes de suelos, obtenidos del Atlas Digital de Suelos de la República Argentina (INTA-AEROTERRA 1995). Sobre el mapa de tipos de suelos se realizó un procedimiento de conversión de mapas vectoriales, del Atlas descripto, a un formato raster con una resolución de 30 segundos.

Luego de georeferenciar los mapas se crearon nuevas coberturas, donde se digitalizaron los puntos correspondientes a cada uno de los pozos y sus valores de profundidad de la napa freática para ambas provincias, y se unieron a los ya facilitados de Mendoza y San Juan. Con el mapa de puntos completo se creó otro de isolíneas de profundidad de freática, y luego se lo transformó a raster, con la misma resolución de las otras variables.

Se analizó la multicolinealidad de las variables (Mac Nally 2000; Olden \& Jackson 2000) y se seleccionaron aquellas que presentaron valores de correlación $\mathrm{r}$ de Pearson inferiores a 0.5 (Mac Nally 2002). De esa manera se eliminó la variable Bio 19 (precipitación del trimestre más frío) por estar correlacionada con la variable Bio 12 (precipitación anual). Esta última se mantuvo en el modelo porque aportaba más información al modelo de acuerdo a los primeros ensayos.

\section{Obtención del modelo y correcciones}

Para generar el modelo final con las variables elegidas se realizaron 100 corridas para obtener un promedio de la probabilidad de ocurrencia de la especie para cada píxel. Los datos fueron divididos al azar, y en cada corrida se seleccionó el $70 \%$ de los datos para generar el modelo y el $30 \%$ para ponerlo a prueba. Para la evaluación del modelo se utilizó el valor promedio del área bajo la curva ROC (AUC por sus siglas en inglés), proporcionada por Maxent, que representa la proporción de predicciones correctamente clasificadas para diferentes umbrales de probabilidad. Como regla general, los modelos con valores de AUC por encima de 0.75 son considerados aceptables y con suficiente capacidad discriminativa (Elith et al. 2006).

Debido a que la presencia de autocorrelación espacial en los datos puede producir resultados sesgados en el modelado de distribución potencial (Dormann et al. 2007; Veloz 2009), evaluamos su existencia a través del coeficiente I de Moran siguiendo la metodología propuesta por De Marco et al. (2008). Dado que en el primer análisis de Maxent la autocorrelación espacial fue alta y significativa $(0.882 ; P=0.005)$, en los residuales del modelo se obtuvieron filtros espaciales para incluirlos en el modelado con el fin de disminuirla hasta valores no significativos. A partir de la herramienta Spatial EigenVector Mapping (SEVM) del programa SAM v.4.0 (Rangel et. al. 2010; http:/ /www.ecoevol.ufg.br/sam) se obtuvieron 12 filtros espaciales que arrojaron autocorrelación espacial significativa y que fueron incluidos al modelo de distribución potencial. Una vez realizadas nuevamente 100 repeticiones se observó una reducción en los valores I de Moran, calculados sobre los residuales del modelo, que no fueron significativos $(0.27, P>0.05)$. Esto indica que se logró disminuir los problemas derivados de la autocorrelación espacial hasta niveles que no implican un sesgo en los resultados del modelo.

\section{Resultados}

\section{Evaluación del modelo}

El promedio de los valores del área bajo la curva (AUC) para los datos de prueba fue 0.946 , con una desviación estándar de 0.004 , lo que indica una predicción mayor que la esperada por azar $(\mathrm{AUC}=0.5)$ y una discriminación bastante precisa de los datos. Para establecer el umbral de probabilidad, sobre el cual se consideró cierta la presencia de bosque, se aplicó como umbral de corte el mínimo de presencia de los puntos de entre- 
namiento (minimum training presence logistic threshold); el umbral fue 0.0428 .

A su vez, sobre dicho umbral existen distintos niveles de probabilidad de ocurrencia de bosque: baja (0.0428-0.2511), media (0.2511$0.517)$ y alta (0.517-1). Estos valores fueron establecidos en función del umbral que marca el percentil 10 de los puntos de entrenamiento, con un valor de 0.2511 (10 percentile training presence logistic threshold), y el umbral establecido a partir del equilibrio entre la omisión de los puntos de entrenamiento y del área prevista (balance training omission, predicted area and threshold value area), que fue 0.517.
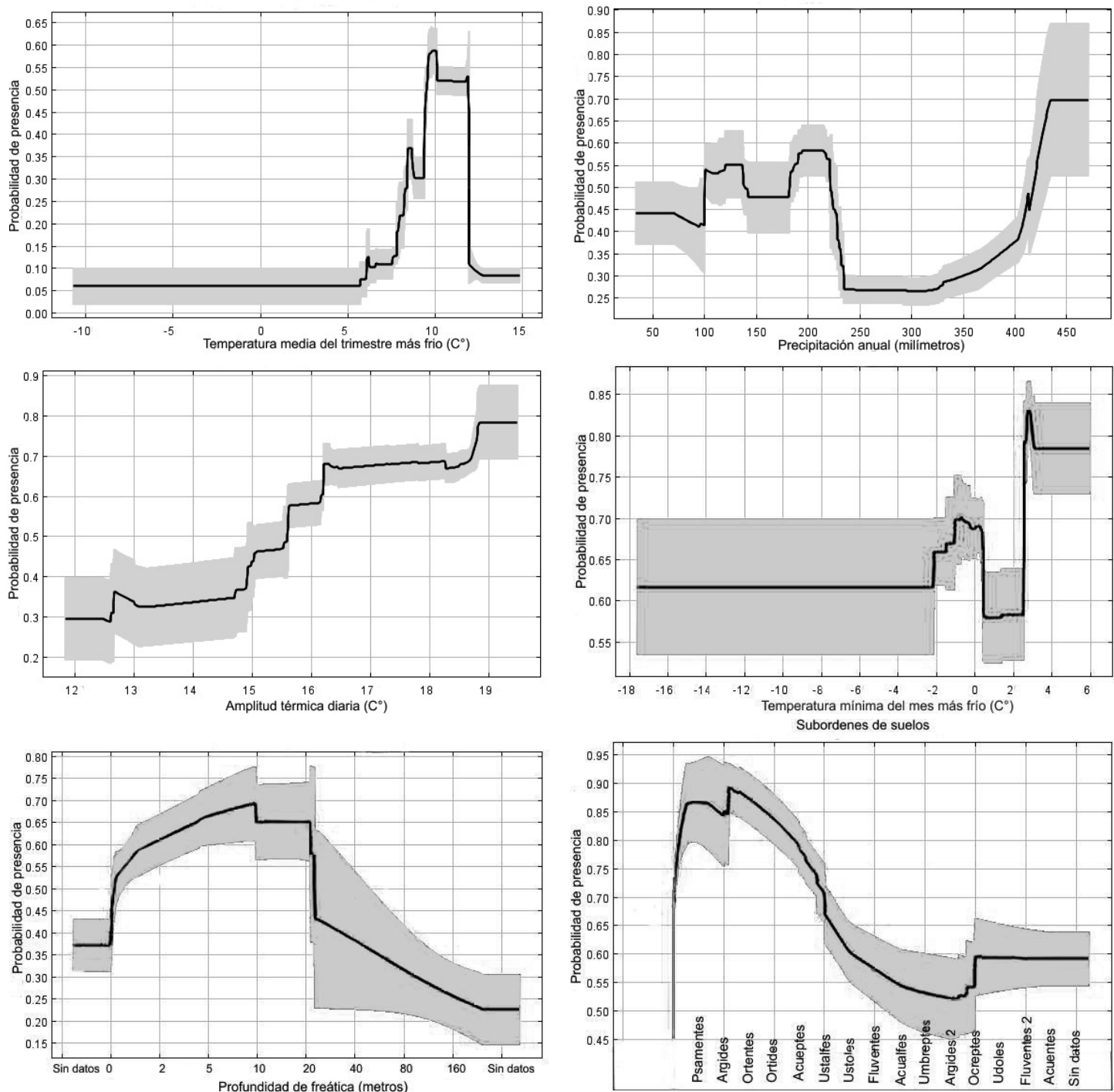

Figura 2. Curvas de respuesta. Estas curvas muestran cómo afecta cada variable ambiental a la predicción de Maxent (el área gris representa el intervalo de confianza). Las curvas muestran cómo cambia la predicción logística cuando se analiza la variación de cada variable ambiental, manteniendo el resto de variables en su valor promedio de la muestra.

Figure 2. Response curves. These curves show how each environmental variable affects the Maxent prediction (grey area is the confidence interval). The curves show how the logistic prediction changes as each environmental variable is varied, keeping all other environmental variables at their average sample value. 
total del modelo, las variables temperatura media del trimestre más frío seguida de temperatura mínima del mes más frío, fueron las más importantes en términos de la ganancia producida en los modelos con sólo una variable. El análisis de las curvas de respuesta de cada variable por separado sobre la predicción de Maxent mostró que la probabilidad aumenta drásticamente cuando la temperatura media del trimestre más frío se ubica entre $5^{\circ} \mathrm{C}$ y $12^{\circ} \mathrm{C}$. Algo similar sucede con la temperatura mínima del mes más frío: cuando dicho valor es mayor a los $-2^{\circ} \mathrm{C}$ la probabilidad crece de forma importante. Por su parte, la precipitación anual muestra una influencia en el aumento de la probabilidad cuando supera los 100 mm/año, y mucho más cuando supera los $400 \mathrm{~mm}$ / año en el límite del Monte con la región Chaqueña. Al analizar la amplitud térmica diaria se observa que entre $16^{\circ} \mathrm{C}$ y $19^{\circ} \mathrm{C}$ las probabilidades de encontrar bosque son mayores. La profundidad de la napa freática presentó sus mayores aportes cuando se encontró a menos de $20 \mathrm{~m}$ de profundidad (Figura 2) y fue la segunda variable con más importancia exclusiva (mostrada en la diferencia de explicación al modelo cuando está presentes o ausente) después de la temperatura media del trimestre más frío (Tabla 1). Por lo tanto, se comportó como una limitante muy importante para la distribución del bosque.

La variable categórica suelos fue la segunda que más contribuyó y tuvo una alta importancia exclusiva. Los subórdenes de suelo que mostraron más influencia positiva en el modelo fueron los ortentes, psammentes (entisoles), argides y ortides (aridisoles). En menor medida se encontró una influencia de los suelos ustalfes (alfisoles) y acueptes (inceptisoles) sobre el aumento de la probabilidad (Figura 2).

\section{Mapa de distribución potencial}

El mapa resultante de las cien corridas muestra zonas donde el modelo predice alta probabilidad que coinciden con una importante existencia de bosques actuales (Figura 1) (e.g., Telteca, Villa Unión, Fiambalá, Nacuñán). Por otro lado, también muestra áreas con una alta probabilidad media que no se corresponde con la baja presencia actual, como el caso de Bermejo, el norte de Pipanaco, o sí concuerda con amplios sectores de bosques poco densos y dispersos, como sucede en torno a Chilecito. En la zona de General Alvear, el modelo mostró baja probabilidad de presencia de bosque en algunos sectores, aunque se conoce de la existencia actual e histórica de bosque. No obstante, dichos algarrobales presentan particularidades en su estructura, más similares a los bosques del Monte austral, con formas más arbustivas.

\section{DisCUSIÓN}

En este trabajo utilizamos los algoritmos del modelado por máxima entropía con el propósito de modelar la distribución potencial de los bosques de P. flexuosa. Esto implica un subconjunto del área de distribución de la especie en el que las condiciones ambientales permiten el desarrollo de esta especie en forma arbórea, con una densidad mayor a 20 árboles/ha y una cobertura mayor a $15 \%$. Hasta el momento, la mayor parte de los trabajos que utilizan este tipo de modelos se centran en la evaluación de la distribución potencial de especies individuales. Sin embargo, recientemente se ha comenzado a utilizar para el análisis y mapeo de distribución potencial de formaciones vegetales (Tarkesh \& Jetschke 2012).

El modelo de distribución muestra una mayor probabilidad de ocurrencia de bosques en zonas donde se encuentran áreas boscosas actuales, conocidas y descriptas en distintos trabajos (Vervoorst 1954; Morello 1958; Abraham \& Prieto 1999; Villagra \& Villalba 2001; Prieto et al. 2003; Villagra et al. 2004; 2005; Alvarez et al. 2006). Sin embargo, también encontramos zonas con alta probabilidad de ocurrencia de algarrobales donde los relevamientos de campo demuestran que la presencia de bosque no es muy abundante en la actualidad (Rojas 2013a; Alvarez et al. 2006; Gil 2013; Villagra et al. datos no publicados). En alguna de estas áreas se ha documentado la presencia de bosques hacia mediados de siglo XIX (Rojas 2013a). Esto sugiere que la diferencia entre áreas potenciales y áreas de presencia documentada se podrían explicar por impactos antrópicos que habrían producido una disminución de la cobertura boscosa. Ejemplos de estos casos serían algunas áreas del norte de Pipanaco (incluyendo sectores de Bañado de los Pantanos, Termas de Santa Teresita), sectores de Chilecito y Bermejo y departamento de Jáchal (Rojas 2013a; Gil 2013), áreas donde el modelo predice una alta probabilidad de ocurrencia y en las que existen registros históricos de importante presencia de bosque (Schickendantz \& Lafone Quevedo 1881; Viteau 1910; Pardo 1960; Rojas 2013a) aunque, en muchos casos, la cobertura arbórea 
actual no es suficiente para ser considerado un bosque y en cambio existen zonas de arbustales o peladales intercaladas con árboles (Rojas 2013a).

En zonas donde se observa alta coincidencia entre lo potencial y lo muestreado en los últimos años, es probable que los impactos hayan sido menores o más recientes (Telteca, Villa Unión, Fiambalá-Tinogasta) o existan tasas elevadas de recuperación (Ñacuñán, este y oeste de Pipanaco, este de Chilecito y Vinchina) (Claver \& Roig-Juñent 2001; Villagra et al. 2005; 2009). Son pocas las zonas en que el modelo presentó bajas probabilidades de encontrar bosque y se conoce la existencia de bosques actualmente. Estos casos se podrían explicar por el efecto de otras variables no consideradas en el modelo, como pueden ser el efecto de incendios o la carga ganadera. Estas últimas variables, más algunas inconsistencias del modelo al trabajar rangos latitudinales tan amplios, podrían ser las causantes de que en General Alvear (Mendoza) el modelo prediga baja probabilidad pero existan algarrobales importantes (Cesca 2011).

Según el procedimiento Jackknife, la temperatura media del trimestre más frío es la variable que más influye en la distribución de los bosques. Esta variable estaría relacionada con los periodos de heladas que pueden afectar el crecimiento de la especie debido a daños producidos sobre la yema apical (Fisher 1977). La temperatura media del trimestre más frío es importante destacar que aporta mucho más en comparación con la precipitación anual; es decir que la presencia de agua subterránea cercana a la superficie determinaría la existencia de bosques sobre todo en zonas en donde la precipitación es más escasa, como sucede en el norte hasta la zona de Telteca. Esto coincide con lo propuesto por Morello (1958), quien afirma que en el Monte, los bosques de P. flexuosa no pueden crecer independientemente de una capa de freática accesible en zonas donde la precipitación es menor a $350 \mathrm{~mm} /$ año. Se ha demostrado el consumo de agua freática por esta especie mediante de distintas aproximaciones hidrológicas (Jobbágy et al. 2011). Sin embargo, también esta especie puede sobrevivir usando agua superficial cuando la freática no está accesible, aunque el crecimiento es menor y no alcanza formas arbóreas; por esta razón no forma bosques de las características que consideramos en este trabajo (Guevara et al. 2010; Villagra et al. 2011). Estos datos sugieren que la distribución de los bosques de $P$. flexuosa es un subconjunto de la distribución de la especie que presenta las condiciones que le permiten alcanzar la forma arbórea, como la existencia de agua freática y temperaturas mínimas tales que no afecten el desarrollo de los individuos. Por su parte, la especie puede extender su distribución a áreas subóptimas, con menor acceso a agua subsuperficial, en forma de individuos aislados o de menor desarrollo. Por ejemplo, en el Monte Austral se han encontrado formaciones arbustivas de esta especie, posiblemente controladas por algún factor que se encuentra fuera del óptimo, como la temperatura (Cony 1993).

En el caso de los subórdenes de suelos, las regiones con mayor probabilidad para la ocurrencia de bosques coinciden con aridisoles y entisoles, suelos con poco desarrollo de horizontes y materia orgánica. Ellos aumentan la probabilidad de existencia en el modelo cuando analiza a los suelos psammentes (arenosos) presentes en todo el Monte, en especial en San Juan y Mendoza, y los ortentes (muy erosionados) que encontramos en Mendoza especialmente en Nacuñan y en gran parte del monte desde Catamarca hasta San Juan.

En menor medida, se encontró que en algunas áreas del norte del área de estudio, los suelos ustalfes (alfisoles) y acueptes (inceptisoles) presentaron una relación con el aumento de la probabilidad de presencia de bosque (Figura 2). Hay que destacar que suelos como los ustalfes son típicos de ambientes semi-áridos del NOA. Por su parte, los acueptes se localizan en áreas planas, deprimidas e inundables, con drenaje pobre y donde la capa de agua freática es cercana a la superficie (lugares muy aptos para el desarrollo del bosque de Prosopis). Según estudios realizados al respecto, el tipo de suelo y su textura son de importancia en el establecimiento y crecimiento de los árboles debido a que controlan la disponibilidad del recurso hídrico (Noy-Meir 1973) y el efecto de otros factores limitantes como la salinidad (Villagra \& Cavagnaro 2005). Además, influyen en la penetración de la raíz (Brar \& Palazzo 1995) y en la absorción y acumulación de dióxido de carbono y formación de biomasa (Villagra \& Cavagnaro 2000; Vilela \& Ravetta 2001).

Finalmente, si se tiene en cuenta el marco de la Ley de Bosques Nativos (Ley 26331/07), este trabajo puede ser muy útil para dirigir los esfuerzos de muestreo a sitios que han sido poco relevados y que muestran alta 
probabilidad de ocurrencia de bosques de $P$. flexuosa en el Monte (como Bermejo y La Paz). Al combinar la información de distribución potencial con datos históricos de distribución de bosques y de uso, es posible priorizar las zonas de análisis para determinar áreas de conservación y de recuperación (como Pipanaco, Chilecito y Fiambalá). Si bien este estudio no analiza la influencia de escenarios climáticos futuros, en el desarrollo del bosque nativo sería de gran interés evaluar dichos condicionantes en contextos de probable variabilidad climática (Boulanger et al. 2007; Labraga \& Villalba 2009).

Agradecimientos: A Guillermo Debandi por sus numerosos y valiosos aportes para el desarrollo del presente estudio. Este trabajo fue financiado por CONICET, la Agencia Nacional de Promoción Científica y Tecnológica de Argentina y la Universidad Nacional de Cuyo.

\section{BIBLIOGRAFÍA}

Abraham, ME \& MR Prieto. 1999. Vitivinicultura y desertificación en Mendoza. En: García Martínez, B (ed.). Estudios de historia y ambiente en América: Argentina, Bolivia, México, Paraguay. IPGH - Colegio de México. México.

Alsos, IG; T Alm; S Normand \& C Brochmann. 2009. Past and future range shifts and loss of diversity in dwarf willow (Salix herbacea L.) inferred from genetics, fossils and modeling. Global Ecology and Biogeography, 18:223-239.

Alvarez, JA; PE Villagra; MA Cony; EM Cesca \& JA BONINSEGNA. 2006. Estructura y estado de conservación de los bosques de Prosopisflexuosa D.C. (Fabaceae, subfamilia: Mimosoideae) en el noreste de Mendoza (Argentina). Revista Chilena de Historia Natural, 79:75-87.

Anderson, RP; AT Peterson \& M Gómez-La Verde. 2002. Using niche-based GIS modeling to test geographic predictions of competitive exclusion and competitive release in South American pocket mice. Oikos, 98:3-16.

Asociación Argentina de amigos de la Universidad Ben GURION. 2006. Estudio de Relevamiento y Evaluación de los Recursos Hídricos en Zonas Áridas de la Provincia de Catamarca. Programa de Servicios Agrícolas Provinciales (PROSAP). Programación del Desarrollo Agropecuario (EPDA). Contratación No A-10-S-01/05. Informe Final. Buenos Aires, noviembre 2006.

Boulanger, JP; F Martínez \& EC Segura. 2007. Projection of future climate change conditions using IPCC simulations, neural network sand Bayesian statistics. Part 2: Precipitation mean state and seasonal cycle in South America. Climate Dynamics, 28:255-271.

BRAR, GS \& AJ PaLazzo. 1995. Shoot a roof development of tall and hard fescues in two different soils. J. Environ. Qual., 24:771-781.

CABRera, AL. 1976. Regiones Fitogeográficas Argentinas. En: W.F. Kugler (ed.). Enciclopedia Argentina de Agricultura y Jardinería. Tomo 2(1):85. Buenos Aires.

Cesca, EM; PE Villagra; JA Álvarez \& MA Cony. 2004. Estructura de los algarrobales en una zona afectada por incendios del sudeste de Mendoza. II Reunión Binacional de Ecología, XXI Reunión de la Sociedad Argentina de
Ecología, XI Reunión de la Sociedad Chilena de Ecología. Mendoza.

Cesca, EM. 2011. Influencia del fuego en la estructura y dinámica de los algarrobales del sudeste de Mendoza. Tesis Doctoral. Universidad Nacional de Córdoba. Córdoba. Pp. 132.

Claver, S \& SA Roig-Juñent (eds.). 2001. El desierto del Monte: La Reserva de Biosfera de Ñacuñán. IADIZA - MAB - UNESCO. Mendoza. Pp. 226.

Cony, M. 1993. Programa de Conservación y Mejoramiento de Especies del Género Prosopis en la Provincia Fitogeográfica del Monte, Argentina. Pp.: 37-72 en: IADIZA (ed.). Contribuciones Mendocinas a la Quinta Reunión de Regional para América Latina y el Caribe de la Red de Forestación del CIID. Conservación y Mejoramiento de Especies del Género Prosopis. Convenio CIID-IADIZA. IADIZA-CRICYT-CIID, Mendoza.

Corbalán, V \& G Debandi. 2007. La Lacertofauna de Mendoza: Lista Actualizada, Distribución y Riqueza. Instituto Argentino de Investigaciones de las Zonas Áridas (IADIZA-CONICET). Cuad. herpetol., 21:75-82.

De Marco, P; JA Felizola Diniz-Filho \& LM Bini. 2008. Spatial Analysis improves species distribution modelling during range expansion. Biol. Lett., 4:577-580.

Dormann, CF; JM McPherson; M B Araújo; R Bivand; J Bolliger; ET AL. 2007. Methods to account for spatial autocorrelation in the analysis of species distributional data: a review. Ecography, 30:609-628.

DunCAN, RP; P CASSEY \& TM BLACKBURN. 2009. Do climate envelope models transfer? A manipulative test using dung beetle introductions. Proceedings of the Royal Society B., 267:1449-1457.

Elith, J; SJ Phillips; T Hastie; M Dudík; YE Chee1; et Al. 2011. A statistical explanation of MaxEnt for ecologists. Diversity and Distributions, 17:43-57.

Elith, J; M Kearney \& SJ Phillips. 2010. The art of modeling range-shifting species. Methods in Ecology and Evolution, 1:330-342.

Eltit, J; CH Graham; RP Anderson; M. Dudik; S Ferrier; ET AL. 2006. Novel methods improve prediction of species' distributions from occurrence data. Ecography, 29:129-151.

Elith, J \& JR LeathWick. 2009. Species distribution models: ecological explanation and prediction across space and time. Annual Review of Ecology, Evolution and Systematics, 40:677-697.

F.A.O. 2011. Situación de los bosques del mundo. Roma.

Ferrer, MS; AE Marvaldi \& MF Tognelli. 2007. First records of three species of Oxycraspedus Kuschel (Coleoptera: Belidae) in Argentina and use of a predictive model to compare their potential distribution with the range of their hostplant, Araucaria araucana. Revista Chilena de Historia Natural, 80:327-333.

Ferrier, S \& A Guisan. 2006. Spatial modelling of biodiversity at the community level. Journal of Applied Ecology, 43:393-404.

Fisher, CE. 1977. Mesquite and modern man in Southwestern North América. Pp. 177-188 en: Simpson, BB (ed.). Mesquite, Its biology in two desert ecosystems. Stroudsburg, Pennsylvania.

Flather, CH. 1996. Fitting Species-Accumulation Functions and Assessing Regional Land Use Impacts on Avian Diversity. Journal of Biogeography, 23:155-168.

GIL, A. 2013. Estructura forestal y estado de conservación de los bosques de Prosopis chilensis y Prosopis flexuosa (algarrobales) de la Depresión del Río Bermejo, noreste de San 
Juan. Tesis de grado en Ingeniería en Recursos Naturales Renovables, Facultad de Ciencias Agrarias, Universidad Nacional de Cuyo. Pp. 110.

GonzÁlez-Loyarte, M; M Menenti;P Weidema;FA Roig \& M BARTON. 2000. Mapping vegetation degradation applying remotely sensed data in the arid zones of Argentina. The northeastern plain of Mendoza. Proceedings of United Nations/International Astronautical Federation Workshop on "Operational Strategy for Sustainable Development using Space". United Nations Committee on the Peaceful Uses of Outer Space and Office for Outer Space Affairs, Sao José dos Campos, Brazil. Pp. 175.

Grau, R; NI Gasparri; M Morales; A Grau; E Aráoz; et al. 2007. Regeneración ambiental en el noroeste Argentino. Ciencia Hoy, 17:46-60.

Guevara, A; E González Sosa; HS Azpiri; G Malda BARRERA; M MARTíNEZ; ET AL. 2008. Distribución potencial de algunas leguminosas arbustivas en el Altiplano Central de México. Agrociencia, 42:703-716.

Guevara, A; CV Giordano; J Aranibar; M Quiroga \& PE Villagra. 2010. Phenotypic plasticity of the coarse root system of Prosopis flexuosa, a phreatophyte tree, in the Monte Desert (Argentina). Plant Soil, 330:447-464.

Guisan, A \& NE Zimmermann. 2000. Predictive habitat distribution models in ecology. Ecol. Model., 135:147-86.

Guisan, A \& W Thuillier. 2005. Predicting species distribution: offering more than simple habitat models. Ecology Letters, 8:993-1009.

Haverkost, TR; T SCott; L GARdNER \& A TownSEnd. 2010. Predicción de la distribución de un parásito usando el modelo de nicho ecológico, GARP. Revista Mexicana de Biodiversidad, 81:895- 902.

Hijmans, RJ; SE Cameron; JL Parra; PG Jones \& A Jarvis. 2005. Very High Resolution Interpolated Climate Surfaces for Global Land Areas. International Journal of Climatology, 25:1965-1978.

Hortal, J; JM Lobo \& FM Piera. 2003. Una estrategia para obtener regionalizaciones bióticas fiables a partir de datos Incompletos: El caso de los Escarabeidos (Coleoptera, Scarabaeinae) Ibérico-Baleares. Graellsia, 59(2-3):331-344.

INTA-AEROTERRA. 1995. SIG Atlas de Suelos de la República Argentina. CD-ROM con el programa Arc View incorporado. Instituto de Suelos-CIRN/INTA. Aeroterra S. A., Buenos Aires.

Jayat, JP; SE Pacheco \& PE Ortiz. 2009. A predictive distribution model for Andinomys edax (Rodentia: Cricetidae) in Argentina. Mastozoología Neotropical, 16: 321-332.

JobBÁgy, EG; MD Nosetto; PE VILLAGRa \& RB Jackson. 2011. Water subsidies from mountains to deserts: Their role sustaining groundwater-fed oases in a sandy landscape. Ecological Applications, 21:678-694.

Kearney, M \& WP Porter. 2009. Mechanistic niche modelling: combining physiological and spatial data to predict species' ranges. Ecol. Lett., 12:334-50.

Labraga, JC \& R Villalba. 2009. Climate in the Monte Desert: past trends, present conditions, and future projections. J. Arid Environ., 73:154-163.

LOBO, JM \& FM PIERA. 2002. Searching for a Predictive Model for Species Richness of Iberian Dung Beetle Based on Spatial and Environmental Variables. Conservation Biology, 16(1):158-173.

Manel, S; JM Días; ST Buckton \& SJ Ormerod. 1999. Alternative methods for predicting species distribution: An illustration with Himalayan river birds. Journal of
Applied Ecology, 36:734-747.

Mac Nally, R. 2000. Regression and model-building in conservation biology, biogeography and ecology: the distinction between - and reconciliation of - "predictive" and "explanatory" models. Biodiversity and Conservation, 9:655-671.

Mac Nally, R. 2002. Multiple regression and inference in ecology and conservation biology: further comments on retention of independent variables. Biodiversity and Conservation, 11:1397-1401.

Montenegro, CL; MH Strada; G Parmuchi; E Mangui; et AL. 2007. Informe Sobre Deforestación en Argentina. Unidad de Manejo del Sistema de Evaluación Forestal (UMSEF). Dirección de Bosques y Secretaría de Ambiente y Desarrollo Sustentable de la Nación.

Morello, J. 1958. La Provincia Fitogeográfica del Monte. Opera Lilloana, 2:5-115.

Naoki, K; MI Gómez; RP López; RI Meneses \& J Vargas. 2006. Comparación de modelos de distribución de especies para predecir la distribución potencial de vida silvestre en Bolivia. Ecología en Bolivia, 41:65-78.

Noy-MeIR, I. 1973. Desert Ecosystems: Enviroment and Producers. Annual Review of Ecology and Sistematics, 4:25-51.

NúÑ̃z, MA \& KA MedLey. 2011 Pine invasions: climate predicts invasion success; something else predicts failure. Diversity and Distributions, 17:703-713.

OLDEN, JD \& DA JACKSON. 2000 Torturing data for the sake of generality: how valid are our regression models? Ecoscience, 7:501-510.

PARDO, IL. 1960. La cera de retamo, recurso forestal de la zona árida de la República Argentina. Notas Tecnológicas Forestales, 7:2.

Perosa, M. 2010. Distribución potencial de los bosques de Prosopis flexuosa en la Provincia Biogeográfica del Monte, desde Catamarca hasta Mendoza (Argentina). Tesis de grado en Ingeniería en Recursos Naturales Renovables, Facultad de Ciencias Agrarias, Universidad Nacional de Cuyo. Pp. 127.

PhILlips, SJ; RP ANDERson \& RE SchapiRe. 2006. A maximum entropy modelling of species geographic distributions. Ecological Modelling, 190:231-259.

Piera, FM \& JM Lobo. 2003. Database records as a sampling-effort surrogate to predict spatial distribution of insects in either poorly or unevenly surveyed areas. Acta Entomológica Ibérica e Macaronésica, 1:23-35.

Prieto, MR; PE Villagra; NB Lana \& ME Abraham. 2003. Utilización de documentos históricos en la reconstrucción de la vegetación de la Llanura de la Travesía (Argentina) a principios del siglo XIX. Revista Chilena de Historia Natural, 76:613-622.

RANGel, TF; JAF Diniz-Filho \& LM BinI. 2010. SAM: a comprehensive application for Spatial Analysis in Macroecology. Ecography, 33:46-50.

Richards, CL; BC Carstens \& L Lacey Knowles. 2007. Distribution modelling and statistical phylogeography: an integrative framework for generating and testing alternative biogeographical hypotheses. J. Biogeogr, 34:1833-45.

Rodríguez, J. 1999. Estudio del Subsuelo en el Campo del Arenal. Departamento de Santa María, Provincia de Catamarca. Instituto Nacional del Agua, San Juan.

RoIG, FA. 1985. Árboles y bosques de la Región Árida Centro Oeste de la Argentina (Provincias de Mendoza y San Juan) y sus posibilidades silvícolas. Actas Forestación en Zonas Aridas y Semiáridas, Segundo Encuentro Regional C.I.I.D. América Latina y El Caribe. Santiago, Chile. Pp. 
145-188.

RoIG, FA. 1993. Aportes a la etnobotánica del género Prosopis. Pp. 99-119 en: IADIZA (eds.). Conservación y Mejoramiento de Especies del Género Prosopis. Mendoza, Argentina. IADIZA.

Rojas, F; MR Prieto; J Alvarez \& E Cesca. 2009. Procesos socioeconómicos y territoriales en el uso de los recursos forestales en Mendoza desde fines de siglo XIX hasta mediados del XX. Revista Proyección, 7.

RojAs, F. 2013a. Procesos ambientales: Deforestación y actividades productivas en los valles y bolsones del Oeste de la Rioja y Catamarca desde mediados del siglo XIX. Tesis doctoral en Geografía, Universidad Nacional de Cuyo.

Rossi, BE \& PE Villagra. 2003. Effects of Prosopis flexuosa on soil properties and the spatial pattern of understory species in arid Argentina. Journal of Vegetation Science, 14:543-550.

Schickendantz, F \& S Lafone Quevedo. 1881. Escritos Económicos. Imprenta del Estado, Catamarca.

SCHRÖDER, B. 2008. Challenges of species distribution modeling belowground. J. Plant Nutr. Soil Sci., 171: 325-37.

SHMIDA, A. 1985. Biogeography of the Desert Flora. Pp. 2377 en: Evenari, M; I Noy-Meir; DW Goodall (eds.). Hot Deserts and Arid Shrublands, A. Capítulo 2 Ecosystems of the World 12A. Elsevier, Amsterdam-Oxford-New York- Tokyo.

Sosic, MV. 1971. Descripción Hidrogeológica del Valle de Antinaco. Provincia de La Rioja. Buenos Aires.

SSRH-INA. 2002. Atlas Digital de los Recursos Hídricos Superficiales de la República Argentina. CD-Rom. Subsecretaría de Recursos Hídricos de la NaciónInstituto Nacional del Agua, Buenos Aires.

STAUFFER, DE. 2002. Linking populations and habitats: Where have we been? Where are we going? Pp. 53-61 en: Scott, JM; PJ Heglund; ML Morrison; MG Raphael; WA Wall; et al. (eds.). Predicting Species Occurrences: Issues of Accuracy and Scale, Covelo, CA. Island Press. USA.

TARKesh, M \& G JetschKe. 2012. Comparison of six correlative models in predictive vegetation mapping on a local scale. Environmental and Ecological Statistics, 19:437-457.

TOGNELLI, MF; SA RoIG-JuÑENT; AE MARVALDI; GE FloRes \& JM Lово. 2009. An evaluation of methods for modelling distribution of Patagonian insects. Revista Chilena de Historia Natural, 82:347-360.

Torres, R \& JP JAyat. 2010. Modelos predictivos de distribución para cuatro especies de mamíferos (cingulata, artiodactyla y rodentia) típicas del Chaco en Argentina. Mastozoología. Neotropical, 17:335-352.

VELOZ, SD. 2009 Spatially autocorrelated sampling falsely inflates measures of accuracy for presence-only niche models. Journal of Biogeography, 36:2290-2299.
VERVOORST, F. 1954. Observaciones ecológicas y fitosociológicas en el bosque de algarrobo del Pilciao, Catamarca. Tesis doctoral no publicada. Universidad de Buenos Aires.

Victoria, JA; ET AL. 1995. Estado actual del conocimiento de los recursos hídricos de la Provincia de La Rioja. Centro Regional de Aguas Subterráneas, documento interno DI-241, San Juan.

Vilela, AE \& DA Ravetta. 2001. The effect of radiation on seedling growth and physiology in four species of Prosopis L. (Mimosaceae). J. Arid Environ., 44:415-423.

Villagra, PE; CV Giordano; JA Alvarez; JB Cavagnaro; A GuevarA; ET AL. 2011. Ser planta en el desierto: estrategias de uso de agua y resistencia al estrés hídrico en el Monte Central de Argentina. Ecología Austral, 21:29-42.

Villagra, PE; G Defossé; H Del Valle; MS Tabeni; CM ROSTAGNO; ET AL. 2009. Land use and disturbance effects on the dynamics of natural ecosystems of the Monte Desert. Implications for their management. J. Arid Environ., 73:202-211.

Villagra, PE; JA Boninsegna; JA Alvarez; M Cony; E Cesca; ET AL. 2005. Dendroecology of Prosopis flexuosa woodlands in the Monte desert: Implications for their management. Dendrochronologia, 22:209-213.

Villagra, PE; MA Cony; NG Mantován; BE Rossi; MM GONZÁLEZ; ET AL. 2004. Ecología y manejo de los algarrobales de la Provincia Fitogeográfica del Monte. En: Arturi, MF; JL Frangi \& JF Goya (eds.). Ecología y Manejo de Bosques Nativos de Argentina. Editorial Universidad Nacional de La Plata.

Villagra, PE \& R Villalba. 2001. Estructura poblacional del algarrobal de la Reserva de Ñacuñán. Pp. 71-75 en: Claver, S \& S Roig-Juñent (eds.). El desierto del Monte: La Reserva de Biósfera de Nacuñán. IADIZA - MAB UNESCO. Mendoza.

Villagra, PE \& JB Cavagnaro. 2000. Effects of clayish and sandy soils on the growth of Prosopis argentina and $P$. alpataco seedlings. Ecología Austral, 10:111-119.

Villagra, PE \& JB Cavagnaro. 2005. Effects of salinity-soil type interactions on the establishment, growth and water relations of Prosopis argentina and $P$. alpataco seedlings. Implications for their ecological success. Austral Ecol., 30:325-335.

Villaseñor, JL \& O Téllez-Valdés. 2004. Distribución potencial de las especies del género Jefea (Asteraceae) en México. Serie Botánica, 75:205-220.

VITEAU, P. 1910. Informe sobre el estado de la minería en los distritos mineros de Famatina y Guandacol, de la provincia de la Rioja. División de Minas, Geología e Hidrología. Anales del Ministerio de Agricultura, tomo V, número 1, Buenos Aires.

ZARRILLI, A. 2008. Bosques y agricultura: una mirada a los límites históricos de sustentabilidad de los bosques argentinos en un contexto de la explotación capitalista en el siglo XX. Revista Luna Azul, 26:87-106. 